\title{
Antifreeze proteins: computer simulation studies on the mechanism of ice growth inhibition
}

\begin{abstract}
Hiroki Nada ${ }^{1}$ and Yoshinori Furukawa ${ }^{2}$
Antifreeze proteins (AFPs), which are present in the bodily fluids of organisms inhabiting cold environments, function as inhibitors of ice growth by binding to certain planes of ice crystals. However, the exact mechanism of ice growth inhibition is still poorly understood as it is exceedingly difficult to experimentally analyze the molecular-scale growth kinetics of ice crystals at the planes to which the proteins bind. This review paper focuses on computer simulation studies on the mechanism of ice growth inhibition by AFPs. Recent molecular dynamics simulations of a growing ice-water interface to which protein is bound have indicated that, when the protein is stably bound to the interface, the growth rate of ice surrounding the protein decreases drastically, owing to depression of the ice melting point through the Gibbs-Thomson effect. The observed decrease in growth rate is expected to correspond to ice growth inhibition in real-world systems. In addition to presenting published simulation studies on the mechanism of ice growth inhibition by AFPs, this review also outlines the direction of future simulation studies in this field.
\end{abstract}

Polymer Journal (2012) 44, 690-698; doi:10.1038/pj.2012.13; published online 14 March 2012

Keywords: crystal growth control; ice-binding protein; ice-water interface; molecular dynamics (MD); thermal hysteresis

\section{INTRODUCTION}

Various organisms inhabiting cold environments produce a certain type of protein in their bodies during winter. The proteins are known as antifreeze proteins (AFPs) or antifreeze glycoproteins (AFGPs). ${ }^{1}$ Thus far, these proteins have been found in various species of fish, ${ }^{2-4}$ insects, $^{5-7}$ bacteria $^{8,9}$ and plants ${ }^{10,11}$ (hereafter, AFPs and AFGPs are collectively referred to as AFPs).

AFPs function as inhibitors of ice growth. ${ }^{1}$ Consequently, liquid water does not completely freeze if it contains dissolved AFPs, even at temperatures lower than the melting point $T_{m}$ of ice. AFPs have received considerable attention with regards to freeze tolerance and freeze avoidance in organisms, ${ }^{12}$ food processing, ${ }^{13}$ cryopreservation ${ }^{13}$ and ice slurries. ${ }^{14}$

AFPs are of interest with respect to not only ice crystals, but also other types of crystals. For example, elucidating the mechanism whereby AFPs control ice growth might aid the understanding of the control of biomineral crystal growth by organic molecules, ${ }^{15}$ and might also be useful for developing crystal growth technologies such as crystal morphology engineering, as well as for designing novel composite materials. ${ }^{16}$

A number of experimental studies have been performed to investigate AFPs,, $17-21$ and theoretical models of ice growth inhibition by AFPs have also been proposed. ${ }^{22-25}$ Most of the existing theoretical models assume that ice growth inhibition is induced by strong, stable binding of AFPs to certain planes of ice crystals. The models suggest that the stability of AFP binding to these ice crystal planes and the molecular-scale growth kinetics of ice around AFPs at the planes are vital for understanding the actual mechanism of ice growth inhibition.

To date, the ice crystal planes to which AFPs preferably bind have been experimentally determined for AFPs extracted from a few species. ${ }^{26,27}$ However, the stability of binding to the ice crystal planes remains unclear. Moreover, it is almost impossible to conduct experimental analyses on the molecular-scale growth kinetics of ice around AFPs at the planes. Thus, despite the considerable efforts in this area, our understanding of the mechanism of ice growth inhibition by AFPs is still poor.

Computer simulation based on a stochastic model such as molecular dynamics (MD) or the Monte Carlo method ${ }^{28,29}$ is a powerful tool for analyzing the stability of AFP binding to ice and the molecular-scale growth kinetics of ice. Thus far, several research groups have used computer simulation for investigating the stable binding conformations of AFP at an ice crystal plane. ${ }^{30-37}$ Recently, we also used MD simulation to analyze the molecular-scale growth kinetics at an ice-water interface to which AFP was bound. ${ }^{38,39}$ These computer simulation studies have contributed to the understanding of the molecular-scale mechanism of ice growth inhibition by AFPs. In this review paper, we discuss the recent progress, as well as the future direction, of computer simulation studies on the mechanism of ice growth inhibition by AFPs.

${ }^{1}$ National Institute of Advanced Industrial Science and Technology (AIST), Tsukuba, Japan. and ${ }^{2}$ Institute of Low Temperature Science, Hokkaido University, Sapporo, Japan Correspondence: Dr H Nada, National Institute of Advanced Industrial Science and Technology (AIST), Research Institute for Environmental Management and Technology, 16-1 Onogawa, Tsukuba 305-8569, Japan.

E-mail: hiroki.nada@aist.go.jp

Received 24 November 2011; revised 31 January 2012; accepted 1 February 2012; published online 14 March 2012 


\section{FUNDAMENTALS OF AFPs}

Here, we briefly summarize the fundamentals of AFPs, namely the structure of various AFPs, the concept of thermal hysteresis, ice growth shapes induced by AFPs and theoretical models of ice growth inhibition by AFPs. Details on these topics can be found in other review papers and books. ${ }^{1,17-20,40}$

\section{Structure of AFPs}

AFPs are classified into several types according to the features of their three-dimensional structure (Figure 1). Type-I AFPs have a simple $\alpha$-helical structure. ${ }^{41-43}$ The most well-known type-I AFP is winter flounder AFP (wfAFP). ${ }^{41,42}$ Furthermore, type-II AFPs are cysteinerich disulphide-stabilized globular proteins, ${ }^{44,45}$ type-III AFPs are globular proteins ${ }^{46}$ and type-IV AFPs are $\alpha$-helical proteins rich in glutamine and glutamate. ${ }^{47}$ In contrast, insect AFPs have a $\beta$-helical structure. ${ }^{48,49}$ AFGPs are composed of repeating alanine-alaninethreonine (Ala-Ala-Thr) tripeptide units, and each Thr residue in AFGPs bears a disaccharide residue. ${ }^{50}$ AFGPs are highly flexible, and their three-dimensional structure while bound to various planes of ice crystals appears to be rather different from that in bulk water. ${ }^{51}$

\section{Thermal hysteresis}

Figure 2 shows a schematic image of ice growth inhibition by AFPs, together with examples of ice growth shapes induced by AFPs. Normally, once the nucleation of an ice seed occurs in water at a certain temperature $T$ below $T_{m}$, the ice seed continues to grow, and the entire region of water eventually freezes. However, if AFPs are dissolved in the water, the ice seed growth is inhibited, and therefore the water does not freeze completely. Such inhibition occurs at all $T$ above the freezing temperature $T_{f}$, which is considerably lower than $T_{m}$. In this way, AFPs produce a difference between $T_{f}$ and $T_{m}$, which is denoted as $\Delta T_{m}$; this difference is thermal hysteresis. ${ }^{52}$

Normally, an ice crystal grown from pure water is shaped a thin circular disk or hexagonal dendrite with flat $\{0001\}$ basal plane facets. ${ }^{53}$ However, AFPs dramatically alter the shape of ice crystals through their ability to inhibit ice growth (see Figure 2). This alteration of ice shape reflects the fact that AFPs selectively bind to certain specific planes of a growing ice crystal, and the ice crystal eventually becomes fully covered with such planes. For example, wfAFP binds to $\{20 \overline{2} 1\}$ pyramidal planes, resulting in the formation of hexagonal bipyramidal ice crystals covered with $12\{2021\}$ pyramidal planes. ${ }^{17}$ AFPs from other species of fish also bind to pyramidal planes, where the crystallographic index of the planes depends on the species. In contrast, spruce budworm AFP (sbwAFP) binds to both basal and prismatic planes, resulting in the formation of thick hexagonal plate ice crystals. ${ }^{48}$ Furthermore, tenebrio molitor $\mathrm{AFP}^{49}$ induces the formation of rounded lemon-shaped ice crystals, which is assumed to reflect the binding of tenebrio molitor AFP to multiple planes of the ice crystals. ${ }^{54}$

$\Delta T_{m f}$ is shown as a function of the AFP concentration $c$ for typical species in Figure $3 \mathrm{a}$, where $\Delta T_{m f}$ increases together with $c$. As $\Delta T_{m f}$ for insect AFPs is much larger than that of fish AFPs, insect AFPs are

\begin{tabular}{|c|c|c|c|c|}
\hline \multicolumn{4}{|c|}{ Fishes } & Insects \\
\hline AFP (Type 1) & AFP (Type 2) & AFP (Type 3) & AFGP & Hyperactive \\
\hline Alanine-rich $\alpha$-helical & $\begin{array}{l}\text { Cystein-rich } \\
\text { globular }\end{array}$ & Globular & $\begin{array}{l}\text { (Alanine- } \\
\text { alanine- } \\
\text { threonine plus } \\
\text { dissaccharide } \\
\text { groups) repeats }\end{array}$ & $\beta$-helical \\
\hline
\end{tabular}

Figure 1 Illustrations of the structure of typical AFPs.

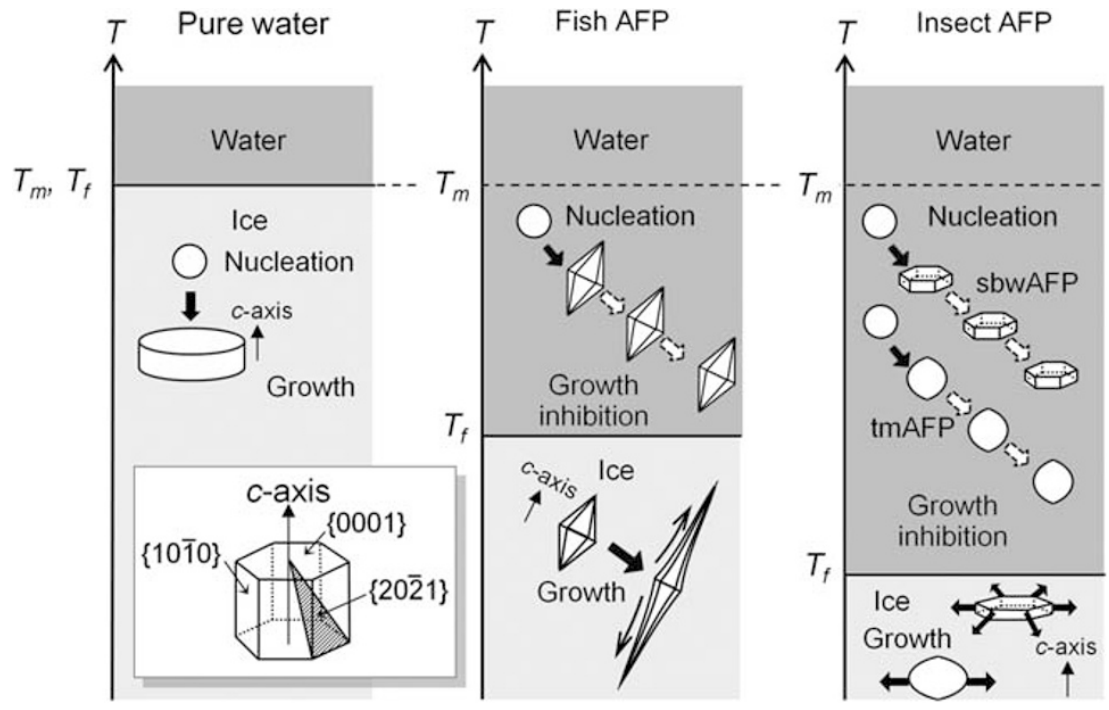

Figure 2 Schematic of the mechanism of ice growth inhibition by AFPs. A full color version of this figure is available at Polymer Journal online. 
a

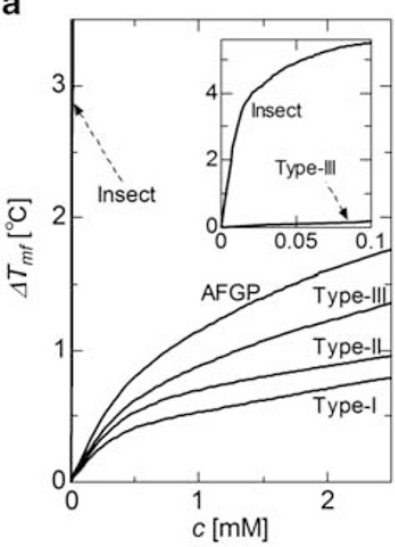

b $T$

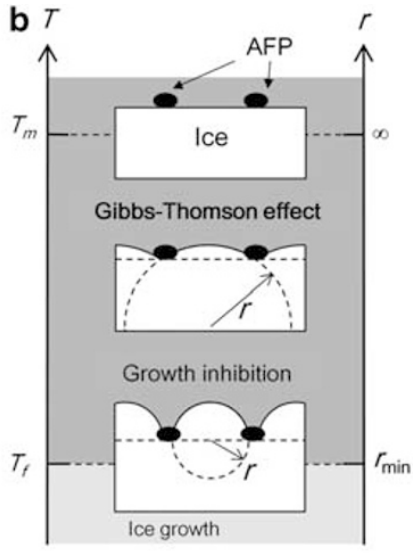

Figure 3 (a) $\Delta T_{m f}$ as a function of $c$ for typical species. Plots for $\Delta T_{m f}$ for wfAFP, 55,56 sea raven AFP, ${ }^{45}$ ocean pout AFP, ${ }^{57}$ arctic cod AFGP50 and sbWAFP57 are shown as representative examples for type-I, -II, -III and insect AFPs, respectively. For AFGP, data on arctic cod AFGP with molecular mass of $28.8 \mathrm{kDa}$ are shown. (b) Schematic of the GibbsThomson model for ice growth inhibition by AFPs. A full color version of this figure is available at Polymer Journal online.

often referred to as hyperactive AFPs. ${ }^{54}$ Note that for all types of AFPs, the decrease in $T_{m}$ caused by thermodynamic factors, such as changes in the free energy of water by mixing AFPs into it, is negligibly small.

\section{Theoretical model of ice growth inhibition}

Thus far, several theoretical models of ice growth inhibition by AFPs have been proposed, ${ }^{22-25}$ the most widely accepted of which is the adsorption-inhibition model. ${ }^{22}$ In this model, ice growth inhibition is explained by assuming irreversibly strong binding of AFPs to certain interfaces of ice crystals. ${ }^{26}$ In other words, when AFP undergoes irreversibly strong binding to an interface of a growing ice crystal, the interface around the AFP is curved (Figure $3 \mathrm{~b}$ ). Consequently, $T_{m}$ at the curved interface becomes locally lower than $T_{m}$ of bulk ice because of the Gibbs-Thomson (or Kelvin) effect. ${ }^{1}$

Assuming the growth of a spherical ice crystal with a radius $r$ around AFPs at an interface of an ice crystal, the depression $\delta T_{m}$ in $T_{m}$ caused by the Gibbs-Thomson effect is given as $\delta T_{m}=2 v \gamma T_{m} / r \Delta H$, where $\gamma$ is the free energy of the interface, $v$ is the molar volume and $\Delta H$ is the heat of crystallization. $\delta T_{m}$ increases as $r$ decreases and reaches a maximum when $r$ decreases to half the distance between two AFP molecules bound to the interface $\left(r_{\min }\right)$; in this case, the shape of the grown ice crystal corresponds to a hemisphere. It is natural to assume that $r_{\min }$ is inversely proportional to $c$. Thus, the maximum of $\delta T_{m}$, which corresponds to $\Delta T_{m f}$, increases together with $c$. Consequently, the model based on the Gibbs-Thomson effect qualitatively explains why ice growth is inhibited by the binding of AFP to an ice interface.

However, the estimated $\delta T_{m}$ from the model appears to be notably larger than $\Delta T_{m f}$ in real-world systems. ${ }^{58}$ Although the model assumes irreversibly strong binding of AFPs to interfaces of ice crystals, the binding in real-world systems might not be as strong. ${ }^{24,25}$ The stability of AFP binding to interfaces of ice crystals and the molecular-scale growth kinetics of ice around AFPs must be elucidated in order to confirm whether the model applies to realworld systems, and hence these two parameters are crucial to the understanding of the mechanism of ice growth inhibition by AFPs in real-world systems. Unfortunately, analyzing these factors with
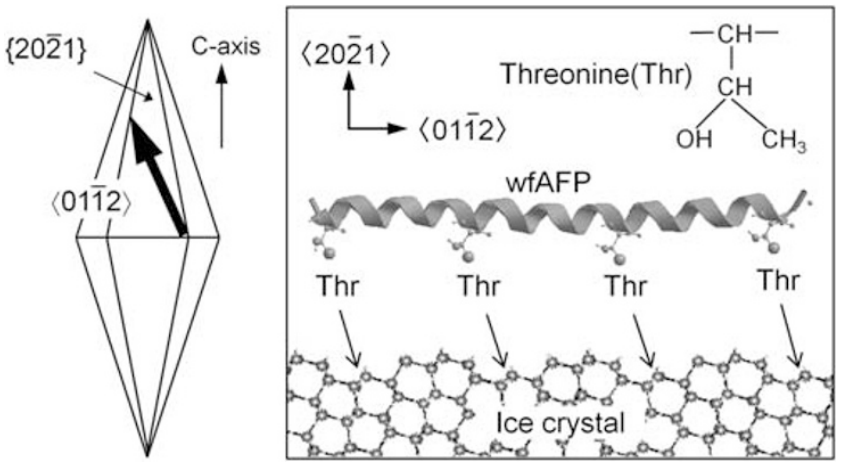

Figure 4 Schematic representing the features of the most energetically stable binding conformation of wfAFP at the ideal pyramidal plane surface. A full color version of this figure is available at Polymer Journal online.

existing experimental techniques and equipment is difficult, which leaves computer simulation as a useful tool for analyzing these factors.

\section{COMPUTER SIMULATIONS OF AFP BINDING TO ICE CRYSTALS}

A number of computational studies have been performed with the aim to investigate the stability of AFP binding to ice crystals, with particular focus on wfAFP. Wen and Laursen ${ }^{30}$ investigated the most energetically stable binding conformation of wfAFP at the $\{20 \overline{2} 1\}$ pyramidal plane by searching for the binding conformation that minimizes the potential energy. They concluded that the most energetically stable binding occurs when wfAFP's $\alpha$-helical axis aligns with the $\langle 01 \overline{1} 2\rangle$ vector, which is in agreement with the binding conformation predicted by Knight et al. ${ }^{26}$ (Figure 4). Madura et al. ${ }^{31}$ searched for the most energetically stable binding conformation at the pyramidal plane for both the D- and the L-form of wfAFP. Furthermore, Chen and $\mathrm{Merz}^{32}$ used MD simulation to examine the effect of liquid water on the binding conformation of wfAFP at the pyramidal plane. Dalal and Sönnichsen ${ }^{33}$ used Monte Carlo simulation to investigate the most energetically stable binding conformation of wfAFP at both the pyramidal plane and the $\{10 \overline{1} 0\}$ prismatic plane. Jorov et al..$^{34}$ used Monte Carlo simulation to investigate the effect of hydrophobic interactions between wfAFP and the pyramidal plane on the stability of binding. Similarly, computer simulation has been used to investigate energetically stable binding conformations at ice crystal surfaces for shorthorn sculpin type-I AFP, ${ }^{35}$ eelpout type-III AFP, sbwAFP ${ }^{38}$ and tenebrio molitor AFP. ${ }^{59}$

However, in real-world systems, AFPs bind to ice-water interfaces rather than to planes of ice crystals, and AFPs interact strongly not only with ice, but also with water. Moreover, ice-water interfaces have a diffuse structure. ${ }^{60-63}$ Wierzbicki et al. ${ }^{64}$ calculated the free energy of wfAFP adsorption at the ice-water interface of the pyramidal plane in equilibrium. This calculation revealed that rather than binding to ice directly, wfAFP is immersed in the interfacial region, where the structure is an intermediate state between ice and water. Wierzbicki et al. ${ }^{33}$ concluded that binding at the interfacial region becomes most stable when the Thr-Ala-Ala part of wfAFP faces the ice, as opposed to Thr-Ala-Asx in the case of the pyramidal plane.

Consequently, computer simulations for ice crystal planes are not sufficient to elucidate the stability of binding of AFPs to ice in realworld systems. Although several MD simulation studies have focused on AFP binding to an ice-water interface in equilibrium, ${ }^{64-66}$ simulations should also target a growing ice-water interface, as this is precisely where the ice growth inhibition function of AFPs is manifested in real-world systems. 
a

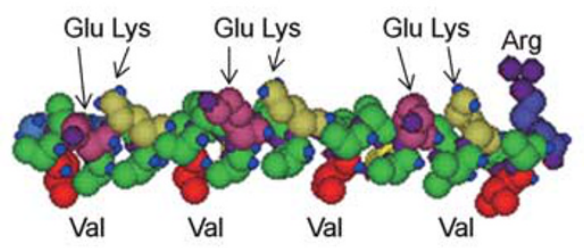

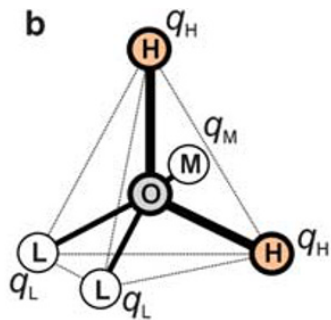

C

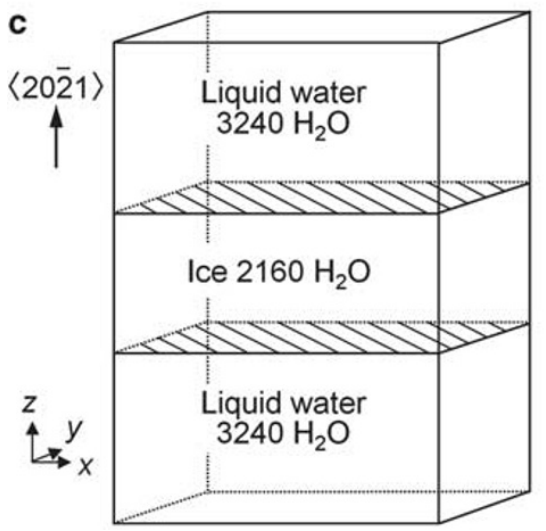

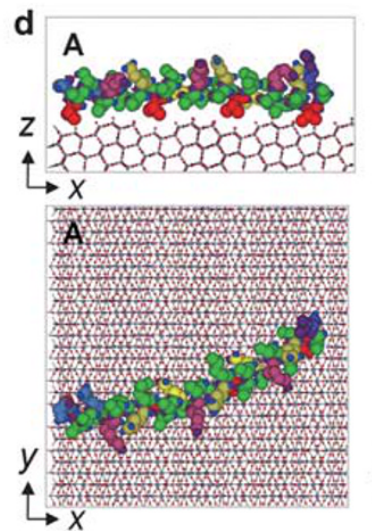

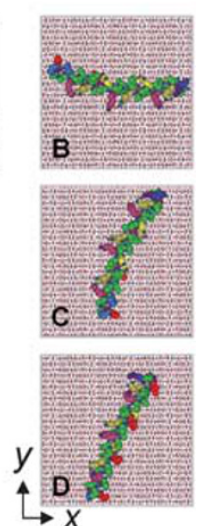

Figure 5 (a) Geometry of water and the positions of interaction sites in the six-site model. ${ }^{67}$ (b) Structure of wfAFP. ${ }^{76}$ (c) A schematic of the simulation system. (d) The initial binding conformations, denoted as A, B, C and D, of AFP at the interface. These initial binding conformations were determined by analyzing the potential energy of the bond between AFP and the ideal pyramidal plane surface. Method used for the analyzing the potential energy can be found in Nada and Furukawa. ${ }^{38}$

\section{SIMULATIONS OF ICE GROWTH INHIBITION BY AFPS}

Recently, we performed MD simulations of growing ice-water interfaces by using a wfAFP mutant bound to the pyramidal plane. ${ }^{38}$ This is the first computer simulation study analyzing the stability of binding of AFP at growing ice-water interfaces and the molecularscale growth kinetics of ice at the interface. An outline of this study is presented below.

\section{MD simulation}

In this study, it was essential to use a water potential model suitable for simulating ice growth. We used a six-site model of rigid water (Figure 5a). ${ }^{67}$ Unlike most of the models commonly used for simulation of water, such as SPC/E, ${ }^{68} \mathrm{TIP} 4 \mathrm{P}^{69}$ and TIP5P, ${ }^{70}$ the sixsite model was proposed for simulation of ice and water near the actual $T_{m}$ at 1 a.t.m.. For such simulations, several MD studies have demonstrated that the six-site model is suitable for simulating ice growth. ${ }^{63,71-73}$ It naturally follows that the six-site model is also suitable for MD simulation of a growing ice-water interface to which AFP is bound.

In this study, we examined a wfAFP mutant, denoted as VVVV2KE, which was synthesized by Haymet et al. ${ }^{74}$ (Figure $5 \mathrm{~b}$ ). In the mutation, the Thr residues of wfAFP are replaced with Val residues, which are strongly hydrophobic (the $\mathrm{OH}$ group of each $\mathrm{Thr}$ residue is replaced with a $\mathrm{CH}_{3}$ group). Moreover, two additional LysGlu salt bridges are introduced in this mutant. The reason for choosing this mutant was that all of its atomic coordinates have been determined precisely in an experimental study, ${ }^{75}$ which facilitated the construction of a rigid model. This mutant also inhibits the growth of ice by binding to the pyramidal plane, ${ }^{76}$ and the stable binding conformation at the pyramidal plane was the same for both the mutant and the original wfAFP. ${ }^{34}$ Therefore, it was reasonable to assume that the mechanism of ice growth inhibition associated with
wfAFP was essentially the same as that associated with the mutant (referred to as AFP below). The potential parameters for AFP were determined in accordance with CHARMM by using the param 19 file for force field calculations. ${ }^{77}$ The LJ parameters for interactions between AFP and water were determined with the LorentzBerthelot rules.

The simulation system was a rectangular parallelepiped in which an ice crystal was sandwiched between two liquid water phases (Figure 5c), and thus the system contained two ice-water interfaces at the $\{20 \overline{2} 1\}$ pyramidal plane. AFP was placed at the surface of each of the two pyramidal interfaces. In this study, four different initial binding conformations of AFP at the interfaces were examined (Figure 5d), where conformation A corresponded to the most energetically stable binding conformation at the ideal pyramidal plane, and conformations $\mathrm{B}, \mathrm{C}$ and $\mathrm{D}$ corresponded to metastable ones. In conformation A, the $\alpha$-helical axis of AFP was aligned along the $\langle 01 \overline{1} 2\rangle$ vector, and each Val residue fit inside the groove at the pyramidal plane (see Figure 5d). Notably, conformation B resembled conformation A whereas conformations $\mathrm{C}$ and $\mathrm{D}$ were vastly different from conformation A.

An MD simulation with a run time covering a period of $4 \mathrm{~ns}$ was conducted by maintaining $T$ and pressure at $268 \mathrm{~K}$ and $1 \mathrm{~atm}$, respectively. Here, $T_{m}$ of ice in the system was estimated to be $278 \mathrm{~K}$. Therefore, $T=268 \mathrm{~K}$ in this simulation corresponded to $-10{ }^{\circ} \mathrm{C}$. Details of the simulation method are given in $\mathrm{Nada}$ and Furukawa. ${ }^{38}$

\section{Growth of ice around AFP}

Figure 6 shows snapshots of AFP and water molecules in a state resembling ice at $4 \mathrm{~ns}$ for all conformations. Water molecules in this state are defined to be connected by hydrogen bonds to each of their four nearest neighbors. The dashed lines show the initial positions of 

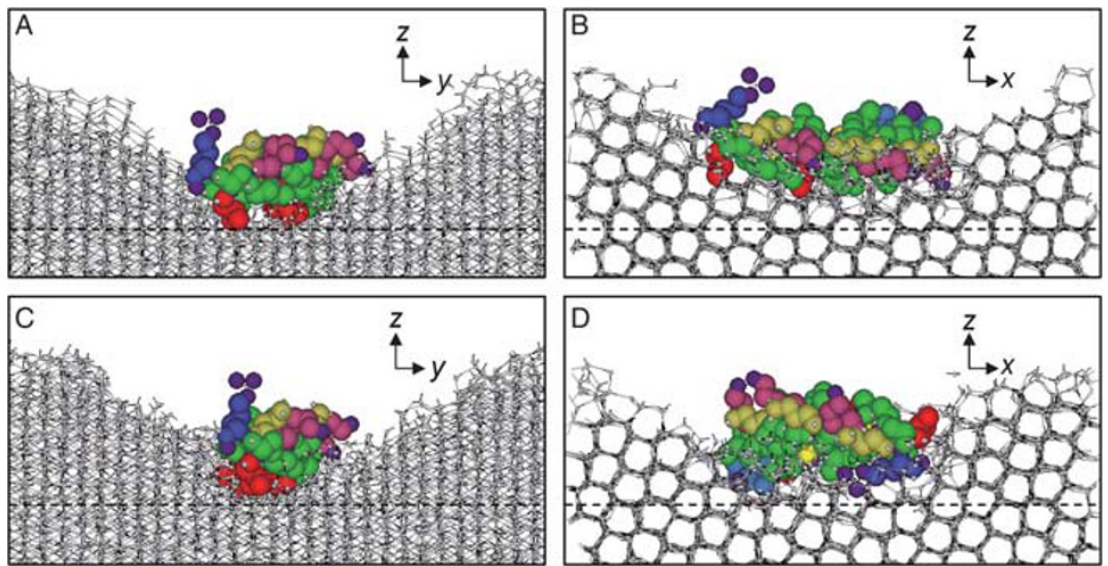

Figure 6 Snapshots of AFP and water molecules forming a structure resembling ice at 4 ns for all conformations. The dashed lines show the initial positions of the interfaces.

the interfaces. Each solid line connecting a pair of water molecules represents a hydrogen bond. It is clear that AFP was partially surrounded by ice that had grown during the simulation. As we describe later, the growth of the surrounding ice had an important role in the stabilization of binding of AFP to the interface.

Figure 7a shows the square displacement $\Delta r_{z}^{2}$ of the center of mass of AFP as a function of time $t$ in the $z$ direction from the beginning of the simulation, and Figure $7 \mathrm{~b}$ shows the shift in rotation angle $\Delta \phi$ around the $\alpha$-helical axis of AFP as a function of $t$. These two panels in the figure reflect the stability of binding of AFP to the interface for each binding conformation. In other words, initial conformation A was stably bound at its initial position during the growth of ice, whereas initial conformation $\mathrm{B}$ became stably bound to the interface only after transforming into conformation A. Initial conformations $\mathrm{C}$ and $\mathrm{D}$ migrated from their initial positions, indicating unstable binding. Thus, conformation A was deemed to be the most stable at the growing pyramidal interface.

\section{Growth rate of ice}

The growth rate $R$ of the ice grown around AFP was estimated for each binding conformation (Figure 8). A large decrease in $R$ occurred during the simulation for conformations $\mathrm{A}$ and $\mathrm{B}$, whereas $R$ for conformations $\mathrm{C}$ and $\mathrm{D}$ was constant over the entire simulation period. Thus, the results indicate that a large decrease in the $R$ occurred only for ice surrounding AFP that was stably bound to the interface, where the observed decrease in $R$ likely corresponded to ice growth inhibition by AFP.

Note that the ice interfaces surrounding AFP for conformations A and $\mathrm{B}$ were curved. Therefore, we suspected that the decrease in $R$ observed for conformations $\mathrm{A}$ and $\mathrm{B}$ originated from a depression in $T_{m}$ at the curved interfaces owing to the Gibbs-Thomson effect. Therefore, using the parameters associated with the Gibbs-Thomson effect, we estimated $\delta T_{m}$ at the curved interface as $5 \mathrm{~K}$ by assuming that ice grown around AFP was in the shape of a column with $r=3.45 \mathrm{~nm}$, which was approximately half of the dimension of the system in the $y$ direction. As $T$ of the system was $-10^{\circ} \mathrm{C}, T$ at the curved interfaces was expected to be $-5^{\circ} \mathrm{C}$.

In order to confirm whether this expectation for the value of $T$ at the curved interfaces was correct, the value of $R$ for ice at $-5^{\circ} \mathrm{C}$ as obtained in the simulation was compared with the value from an experimental study. ${ }^{53}$ The experimentally obtained value of $R$ at $-5^{\circ} \mathrm{C}$ was about $2 \mathrm{~cm} \mathrm{~s}^{-1}$, which is of the same order of magnitude
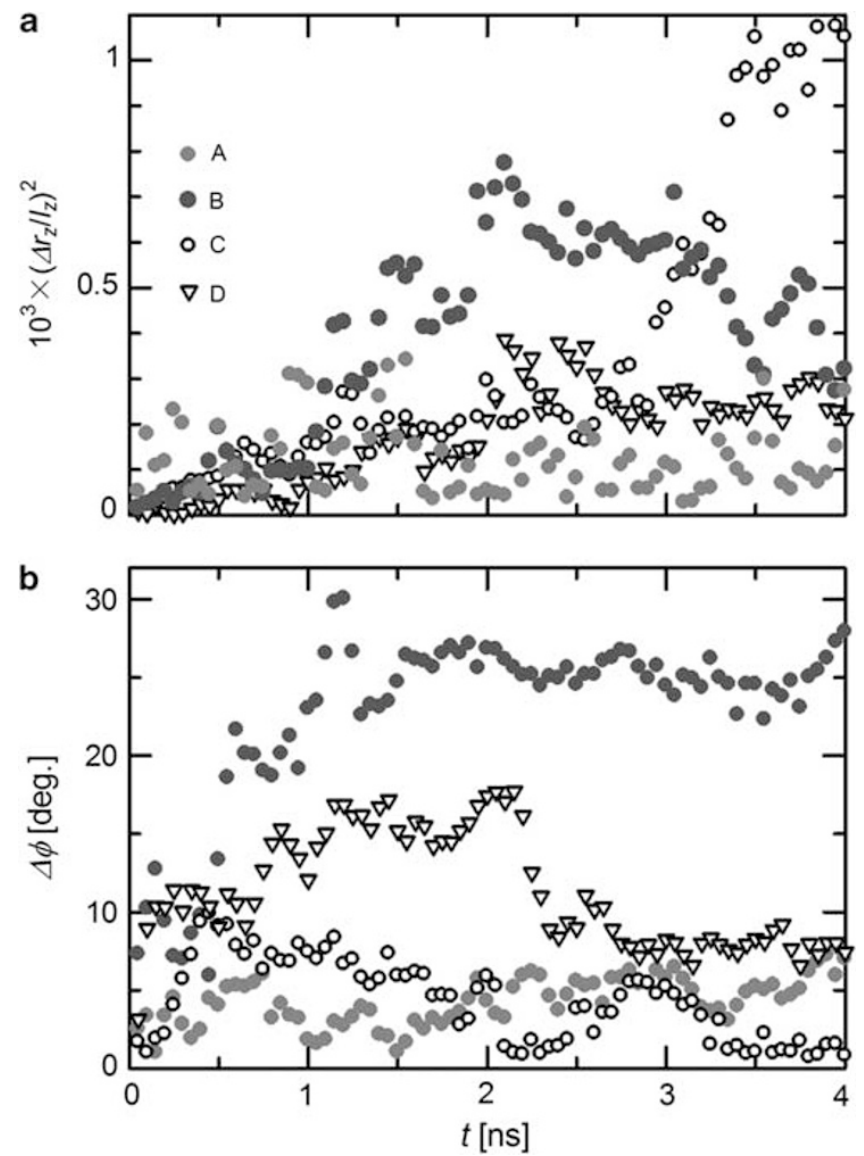

Figure 7 (a) Square displacement $\Delta r_{z}^{2}$ of the center of mass of AFP in the $z$ direction from the beginning of the simulation as a function of time $t$ where $I_{z}$ is the cell length in the $z$ direction. (b) Shifts in the rotation angle $\Delta \phi$ around the $\alpha$-helical axis of AFP as a function of $t$. A full color version of this figure is available at Polymer Journal online.

as that in the period of $2-4 \mathrm{~ns}$ in the simulation $\left(5.9\right.$ and $6.3 \mathrm{~cm} \mathrm{~s}^{-1}$ for conformations $\mathrm{A}$ and $\mathrm{B}$, respectively), which confirms that the observed decrease in $R$ for conformations $\mathrm{A}$ and $\mathrm{B}$ was attributable mainly to a depression in $T_{m}$ at the curved interfaces due to the Gibbs-Thomson effect. The unchanged value of $R$ for conformations $\mathrm{C}$ and $\mathrm{D}$ is attributable to the migration of AFP during ice growth; in 

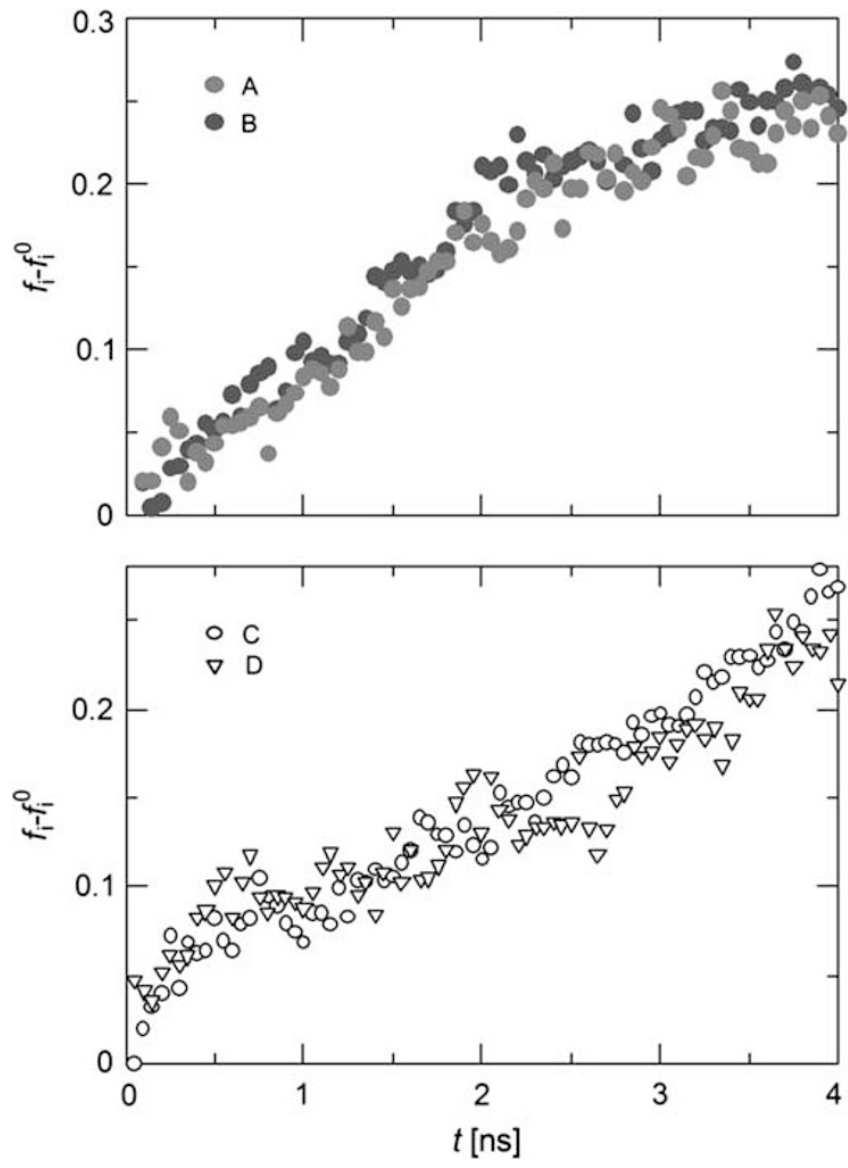

Figure 8 Fraction $f_{\mid L M}$ of ice-like water molecules in the system as a function of $t$. Here, $f_{\mathrm{ILM}}{ }^{0}$ denotes $f_{\mathrm{ILM}}$ at the beginning of the simulation. The increase in $f_{I L M}$ together with $t$ reflects the growth of ice, and the slope of the $f_{\mathrm{ILM}}-t$ relation corresponds to the growth rate $R$. Values of $R$ (in $\mathrm{cms}^{-1}$ ) as estimated from the slopes of the $f_{\mathrm{ILM}}-t$ relations were 19.0 $(0-2 \mathrm{~ns})$ and 5.9 (2-4 ns) for conformation A, 16.9 (0-2 ns) and 6.3 (2-4 ns) for conformation B, 10.4 (0-2 ns) and 14.1 (2-4 ns) for conformation $\mathrm{C}$, and $8.7(0-2 \mathrm{~ns})$ and $12.5(2-4 \mathrm{~ns})$ for conformation D. A full color version of this figure is available at Polymer Journal online.

other words, the migration hindered the formation of a curved interface, and therefore the depression in $T_{m}$ at the ice interface because of the Gibbs-Thomson effect was negligibly small.

\section{Stability of AFP binding at growing interface}

Next, we discuss the observation that binding conformation A was the most stable at the growing pyramidal interface. In binding conformation A, hydrophobic Val residues bound to ice and hydrophilic residues were exposed to the surrounding liquid water. Notably, binding conformation A was the same as the most stable binding conformation at a pyramidal interface in equilibrium as obtained by Jorov et al. ${ }^{34}$, who explained the stability of this conformation as follows. The binding of hydrophobic Val residues to ice produces an entropy gain as compared with the case in which those residues are exposed to liquid water because liquid water molecules surrounding each hydrophobic Val residue tend to form a cage-like structure, causing loss of entropy. Moreover, the exposure of hydrophilic residues to liquid water produces an enthalpy gain as the molecules of liquid water rearrange themselves to form hydrogen bonds with the hydrophilic residues. These entropy and enthalpy gains explain why binding conformation $\mathrm{A}$ was the most stable at the pyramidal interfaces in equilibrium.

To determine whether the explanation for the most stable binding conformation at the pyramidal interface in equilibrium is sufficient for explaining the binding conformation at the growing pyramidal interface, we also performed an MD simulation for the pyramidal interface in equilibrium to which AFP was bound in conformation A. However, stable binding of AFP to the pyramidal interface in equilibrium was not observed. This result indicates that the attraction between AFP and the interface was not sufficient to produce a stable binding conformation. Thus, the above explanation is not sufficient for explaining the most stable binding conformation at growing pyramidal interfaces.

At the growing pyramidal interfaces, AFP was partially surrounded by ice that had grown during the simulation. We found that the absolute value of the attractive force between AFP and the growing ice increased significantly after the growth of the surrounding ice, thus stabilizing the binding of AFP to the pyramidal interface. Thus, we concluded that the growth of ice surrounding AFP was necessary in order to promote stable AFP binding to pyramidal interface and subsequent inhibition of ice growth.

\section{MD simulation for prismatic interfaces}

Investigating the growth kinetics at other ice planes is also important as the formation of a hexagonal bipyramidal ice crystal in the presence of AFP reflects the anisotropy of ice in terms of $R$. Thus, in this study, we also performed an MD simulation for a growing icewater interface in the $\{10 \overline{1} 0\}$ prismatic plane to which AFP was bound. ${ }^{38}$ Two initial binding conformations, namely the most energetically stable conformation and a metastable one at the ideal prismatic plane, were examined as the initial binding conformations at the growing prismatic interface.

In either of the binding conformations, AFP did not bind stably to the prismatic interface (Figure 9a), and $R$ did not change substantially during the simulation (Figure $9 \mathrm{~b}$ ). These results might not be sufficient to conclude whether AFP inhibits the growth of ice at the prismatic interface. However, the results are consistent with the fact that AFP inhibits the growth of ice only at the pyramidal plane in real-world systems. Consequently, we believe that the present MD simulation study provides important results that contribute toward the understanding of the mechanism of ice growth inhibition by AFPs in real-world systems.

However, more detailed studies are necessary in order to clarify certain aspects the mechanism of ice growth inhibition by AFPs. For example, the MD simulation described above was carried out under the assumption that the pyramidal plane existed as a facet plane from the beginning. However, the pyramidal plane appears as a result of growth in real-world systems. ${ }^{77}$ Therefore, it is also important to investigate the mechanism of pyramidal plane formation during the growth of ice crystals in the presence of AFPs.

Notably, the above MD simulation for the prismatic interface indicates a change in the binding conformation of AFP. ${ }^{78}$ Specifically, during the growth of ice at the prismatic interface, AFP molecules gradually tilted in such a way that their $\alpha$-helical axes became parallel to the pyramidal plane (Figure 9c). If the $\alpha$-helical axes of AFP molecules become stably aligned with the $\langle 01 \overline{1} 2\rangle$ vector, a drastic decrease in $R$ might be observed, as in the case of the pyramidal interface. Thus, the observed tilt of the $\alpha$-helical axis of AFP might correspond to the initial step of the process of pyramidal plane formation, which necessitates further inquiry. 


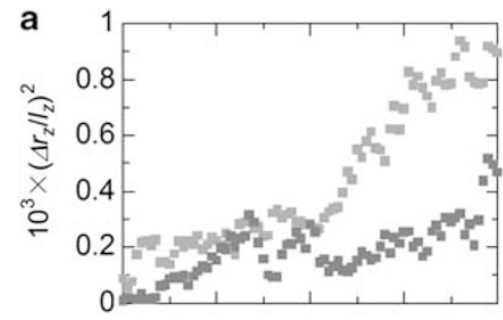

C
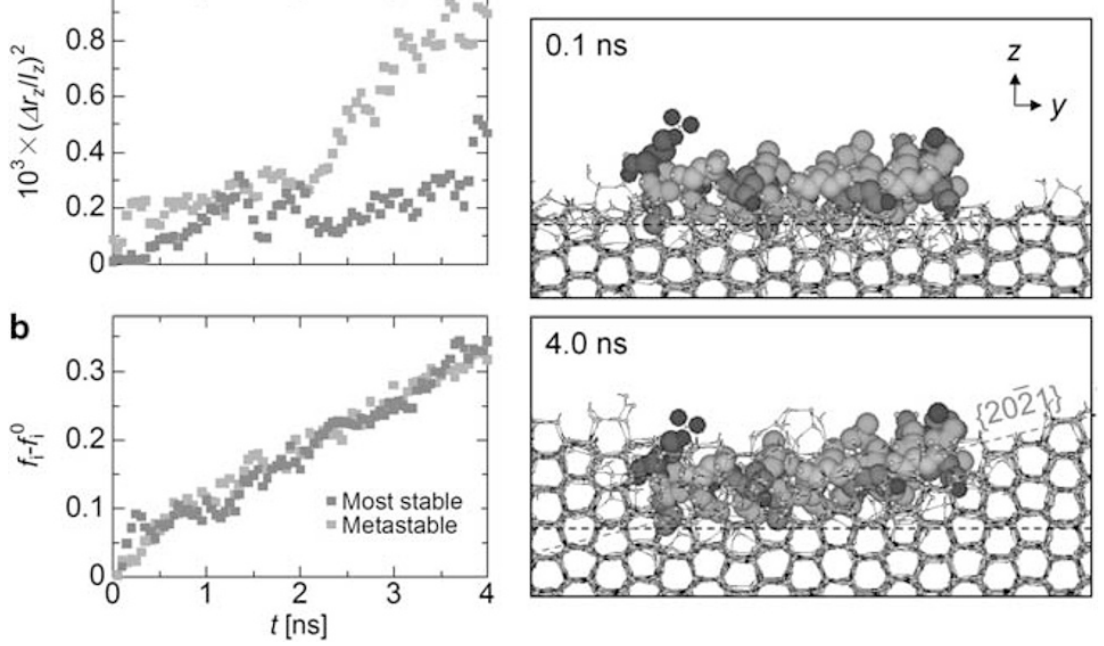

Figure 9 (a) $\Delta r_{z}^{2}$ for the most stable and a metastable binding conformation of AFP as a function of $t$ for the prismatic interface. (b) $f_{\mathrm{ILM}}$ as a function of $t$ for the prismatic interface. (c) Snapshots of the most energetically stable binding conformation of AFP at the ideal prismatic plane and the surrounding structure of water molecules resembling ice at the prismatic interface at 0.1 and $4.0 \mathrm{~ns}$. A full color version of this figure is available at Polymer Journal online.

\section{DISCUSSION}

\section{Simulation methodology}

It is important to establish whether the mechanism of ice growth inhibition proposed on the basis of the MD simulation study using mutant wfAFP also applies to AFPs from other species. Recently, we performed similar MD simulations for a growing ice-water interface of the prismatic plane to which sbwAFP was bound. ${ }^{39}$ As in the case of mutant wfAFP, a large decrease in $R$ was observed for ice grown around sbwAFP that was stably bound to the interface. This finding supports the hypothesis that the decrease in $R$ was attributable to a decrease in $T_{m}$ due to the Gibbs-Thomson effect at the curved interface surrounding sbwAFP. More extensive MD simulation studies on AFPs from other species are necessary in order to determine whether the same mechanism of ice growth inhibition is universal. Nevertheless, the abovementioned MD simulation studies using mutant wfAFP and sbwAFP have reliably demonstrated that molecular processes associated with ice growth inhibition can be directly observed with the aid of MD simulations of a growing ice-water interface to which AFP is bound.

For a thorough understanding of the mechanism of ice growth inhibition by AFPs, it is also important to elucidate the mechanism by which ice crystals undergo a morphological change from a sphere or a circular disk into a polygon covered with specific planes where AFPs inhibit ice growth. However, it has been impossible to observe such morphological changes in the simulations conducted so far due to the use of a directional growth system in which ice growth occurred only for a single crystallographic orientation.

Recently, we also carried out an MD simulation of ice growth at a high pressure by introducing a system in which ice crystals were allowed to grow freely in all directions normal to their $c$-axis (free-growth system), and successfully analyzed the formation mechanism of ice crystals with hexagonal plates. ${ }^{73}$ If a large freegrowth system is used for MD simulation of ice growth in the presence of AFPs, morphological changes induced in crystals by AFPs can be directly observed, thus contributing toward a thorough understanding of the mechanism of ice growth inhibition by AFPs in real-world systems.

\section{Direction of future simulation studies}

Finally, we discuss the direction of future simulation studies on the mechanism of ice growth inhibition by AFPs. One such direction involves simulation studies on hyperactive AFPs. In this regard, Scotter et $a l .{ }^{54}$ have argued that the hyperactivity of certain AFPs stems from their binding to multiple planes of ice crystals, including the basal plane. For example, sbwAFP binds to both prismatic and basal planes of ice. ${ }^{48}$ The question naturally arises as to why hyperactive AFPs bind to multiple planes of ice crystals. Garnham et al. ${ }^{79}$ have suggested that water at the surface of ice crystals to which hyperactive AFPs are bound forms a clathrate-like structure, which can adhere to multiple planes of ice. As computer simulation is a powerful tool for analyzing the structure of water around AFPs, ${ }^{80} \mathrm{MD}$ simulation studies should be performed for multiple planes of ice crystals by considering the structure of water at the surface of ice crystals to which hyperactive AFP is bound. For sbwAFP, we have already performed an MD simulation study for the prismatic plane, ${ }^{39}$ and we intend to extend this study to the basal plane.

Another direction of future work involves the elucidation of the mechanism of ice growth promotion by AFPs. AFPs can promote the growth of ice at planes to which they do not bind. ${ }^{81}$ For example, wfAFP inhibits the growth of ice at pyramidal planes while simultaneously promoting the growth of ice at basal planes, ${ }^{82}$ which is the reason for the formation of hexagonal bipyramidal ice crystals in the presence of wfAFP. Thus, an investigation of the mechanism of ice growth promotion by AFPs is also necessary for a thorough understanding of the mechanism of ice growth inhibition by AFPs in real-world systems. Computer simulation is also expected to serve as a powerful tool for resolving the mechanism of ice growth promotion.

\section{SUMMARY}

In this review, we presented computer simulation studies concerning the mechanism of ice growth inhibition by AFPs. The understanding of this mechanism depends on the elucidation of the stability of AFP binding to various interfaces of ice crystals and the growth kinetics of ice surrounding AFPs at these interfaces. Currently, computer simulation based on intermolecular interaction is the only available tool for analyzing these processes in detail. 
A number of computer simulation studies on the mechanism of ice growth inhibition by AFPs have been carried out to date, most of which have focused on the binding of AFPs to various planes of ice crystals or to ice-water interfaces in equilibrium. Recently, an MD simulation study was carried out with respect to growing ice-water interfaces of the pyramidal plane in the presence of AFPs, ${ }^{38}$ as a result of which a molecular-scale mechanism of ice growth inhibition by AFPs was proposed. A subsequent MD simulation study involving sbwAFP also suggested essentially the same molecular-scale mechanism of ice growth inhibition. ${ }^{39}$ Therefore, simulation studies on AFPs from other species are expected to provide an even deeper understanding of the mechanism of ice growth inhibition, and largescale simulations of free growth systems are expected to contribute toward the elucidating the morphological changes induced in ice crystals by AFPs.

In conclusion, the molecular-scale mechanism of ice growth inhibition by AFPs can be directly analyzed with the aid of computer simulation based on intermolecular interaction. More detailed and extensive computer simulation studies should be conducted for a thorough understanding of the mechanism of ice growth inhibition by AFPs in real-world systems. Similarly, computer simulation is also expected to expand our knowledge regarding the mechanism of crystal growth control by impurities, such as the growth control of mineral crystals by organic molecules. ${ }^{15,16}$

\section{ACKNOWLEDGEMENTS}

This work was supported by a Grant-in-Aid for Scientific Research (C) (No. 21540423) from the Japan Society for the Promotion of Science. Parts of this work, an MD simulation for sbwAFP, were also supported by a Grant-in-Aid for Scientific Research (No. 22107004) on Innovative Areas of 'Fusion Materials' (Area no. 2206) from the Ministry of Education, Culture, Sports, Science and Technology (MEXT).

1 Yeh, Y. \& Feeney, R. E. Antifreeze proteins: structures and mechanisms of function. Chem. Rev. 96, 601-618 (1996).

2 DeVries, A. L., Komatsu, S. K. \& Feeney, R. E. Chemical and physical properties of freezing-point depressing glycoproteins from antarctic fishes. J. Biol. Chem. 245 2901-2908 (1970)

3 Davies, P. L., Hew, C. L. \& Fletcher, G. L. Fish antifreeze proteins: physiology and evolutionary biology. Can. J. Zool. 66, 2611-2617 (1988).

4 Marshall, C. B., Fletcher, G. L. \& Davies, P. L. Hyperactive antifreeze protein in a fish. Nature 429, 153 (2004).

5 Hew, C. L., Kao, M. H., So, Y.-P. \& Lim., K.-P. Presence of cystine-containing antifreeze proteins in the spruce budworm, Choristoneura fumirana. Can. J. Zool. 61, 2324-2328 (1983).

6 Schneppenheim, R. \& Theede, H. Isolation and characterization of freezing-point depressing peptides from larvae of Tenebrio molitor. Comp. Biochem. Phys. $B$. Biochem. Mol. Biol. 67, 561-568 (1980).

7 Duman, J. G., Bennett, V., Sformo, T., Hochstrasser, R. \& Barnes, B. M. Antifreeze proteins in alaskan insects and spiders. J. Insect Physiol. 50, 259-266 (2004).

8 Gilbert, J. A., Christine, P. J., Dodd, E. R. \& Layborn-Parry, J. Demonstration of Antifreeze Protein Activity in Antarctic Lake Bacteria. Microbiology 150, 171-180 (2004).

9 Muryoi, N., Sato, M., Kaneko, S., Kawahara, H., Obata, H., Yaish, M. W. F., Griffith, M. \& Glick, B. R. Cloning and expression of afpA, a gene encoding an antifreeze protein from the arctic plant growth-promoting rhizobacterium Pseudomonas Putida GR12-2. J. Bacteriol. 186, 5661-5671 (2004).

10 Atıcı, Ö. \& Nalbantoğlu, B. Antifreeze proteins in higher plants. Phytochemistry 64, 1187-1196 (2003).

11 Griffith, M. \& Yaish, M. W. F. Antifreeze proteins in overwintering plants: a tale of two activities. Trends Plant Sci. 9, 399-405 (2004).

12 Zachariassen, K. E. \& Kristiansen, E. Ice nucleation and antinucleation in nature. Cryobiology 41, 257-279 (2000).

13 Lee, Jr. R. E., Warren, G. J. \& Gusta, L. V. in Biological Ice Nucleation and Its Applications (APS Press, St Paul, 1997).

14 Grandum, S., Yabe, A., Tanaka, M., Takemura, F. \& Nakamori, K. Characteristics of ice slurry containing antifreeze protein for ice storage applications. J. Thermophys. Heat Transfer 11, 461-466 (1997).

15 Mann, S. Molecular recognition in biomineralization. Nature 332, 119-124 (1998).
16 Sangwal, K. in Additives and Crystallization Processes (John Wiley \& Sons Ltd, Chichester, 2007).

17 Davies, P. L. \& Hew, C. L. Biochemisty of fish antifreeze proteins. FASEB J. 4 2460-2468 (1990).

$18 \mathrm{Hew}$, C. L. \& Yang, D. S. C. Protein interaction with ice. Eur. J. Biochem. 203, 33-42 (1992).

19 Barrett, J. Thermal hysteresis proteins. Int. J. Biochem. Cell Biol. 33, 105-117 (2001).

20 Davies, P. L., Baardsnes, J., Kuiper, M. J. \& Walker, V. K. Structure and function of antifreeze proteins. Phil. Trans. R. Soc. Lond. B. 357, 927-935 (2002).

21 Kristiansen, E. \& Zachariassen, K. E. The mechanism by which fish antifreeze proteins cause thermal hysteresis. Cryobiology 51, 262-280 (2005).

22 Raymond, J. A. \& DeVries, A. L. Adsorption inhibition as a mechanism of freeze resistance in polar fishes. Proc. Natl. Acad. Sci. USA 74, 2589-2593 (1977).

$23 \mathrm{Knight,} \mathrm{C.} \mathrm{A.} \mathrm{\&} \mathrm{DeVries,} \mathrm{A.} \mathrm{L.} \mathrm{Melting} \mathrm{inhibition} \mathrm{and} \mathrm{superheating} \mathrm{of} \mathrm{ice} \mathrm{by} \mathrm{antifreeze}$ glycopeptide. Science 245, 505-507 (1989).

24 Kuroda, T. in Proc. 4th Topical Conference on Crystal Growth Mechanisms 1-4, 1991).

25 Sander, L. M. \& Tkachenko, A. V. Kinetic pinning and biological antifreezes. Phys. Rev. Lett. 93, 128102 (2004).

26 Knight, C. A., Cheng, C. C. \& DeVries, A. L. Adsorption of $\alpha$-helical antifreeze peptides on specific ice crystal surface planes. Biophys. J. 59, 409-418 (1991).

27 Pertaya, N., Marshall, C. B., Celik, Y., Davies, P. L. \& Braslavsky, I. Direct visualization of spruce budworm antifreeze protein interacting with ice crystals: basal plane affinity confers hyperactivity. Biophys. J. 95, 333-341 (2008).

28 Allen, M. P. \& Tildesley, D. J. in Computer Simulation of Liquid (Oxford University Press, Oxford, 1987).

29 Frenkel, D. \& Smit, B. in Understanding Molecular Simulation (Academic Press, San Diego, 1996).

30 Wen, D. \& Laursen, R. A. A. Model for binding of a antifreeze polypeptide to ice. Biophys. J. 63, 1659-1662 (1992).

31 Madura, J. D., Wierzbicki, A., Harrington, J. P., Maughon, R. H., Raymond, J. A. \& Sikes, C. S. Interactions of the $D$ and $L$ forms of winter flounder antifreeze peptide with the $\{201\}$ planes of ice. J. Am. Chem. Soc. 116, 417-418 (1994).

32 Chen, A. \& Merz, K. M. Ice binding mechanism of winter flounder antifreeze proteins. Biophys. J. 73, 2851-2873 (1997)

33 Dalal, P. \& Sönnichsen, F. D. Source of the ice-binding specificity of antifreeze protein type I. J. Chem. Inf. Comput. Sci. 40, 1276-1284 (2000).

34 Jorov, A., Zhorov, B. S. \& Yang, D. S. C. Theoretical study of interaction of winter flounder antifreeze protein with ice. Protein Sci. 13, 1524-1537 (2004).

35 Wierzbicki, A., Taylor, M. S., Knight, C. A., Madura, J. A., Harrington, J. P. \& Sikes, C. $\mathrm{S}$. Analysis of shorthorn sculpin antifreeze protein stereospecific binding to $(2-10)$ faces of ice. Biophys. J. 71, 8-18 (1996).

36 Madura, J. D., Taylor, M. S., Wierzbicki, A., Harrington, J. P. \& Sönnichsen, F. D. The dynamics and binding of a type III antifreeze protein in water and on ice. Theochem. 388, 65-77 (1996).

37 Jia, Z., DeLuca, C. I., Chao, H. \& Davies, P. L. Structural basis for the binding of a globular antifreeze protein to ice. Nature 384, 285-288 (1996).

38 Nada, H. \& Furukawa, Y. Growth inhibition mechanism of an ice-water interface by a mutant of winter flounder antifreeze protein: a molecular dynamics study. J. Phys. Chem. B 112, 7111-7119 (2008).

39 Nada, H. \& Furukawa, Y. Growth inhibition at the ice prismatic plane induced by a spruce budworm antifreeze protein: a molecular dynamics simulation study. Phys. Chem. Chem. Phys. 13, 19936-19942 (2011).

40 Graether, S. P. in Biochemistry and Function of Antifreeze Proteins (ed. Graether, S. P.) Ch. 6, 141-189 (Nova Science Publishers, Inc., New York, 2010).

41 Yang, D. S. C., Sax, M., Chakrabartty, A. \& Hew, C. L. Crystal structure of an antifreeze polypeptide and its mechanistic implications. Nature 333, 232-237 (1988).

42 Sicheri, F. \& Yang, D. S. C. Ice binging structure and mechanism of an antifreeze protein from winter flounder. Nature 374, 427-431 (1995).

43 Kwan, A. H. Y., Fairley, K., Anderberg, P. I., Liew, C. W., Harding, M. M. \& Mackay, J. P. Solution structure of a recombinant type I sculpin antifreeze protein. Biochemistry 44, 1980-1988 (2005).

44 Slaughter, D., Fletcher, G. L., Ananthanarayanan, V. S. \& Hew, C. L. Antifreeze proteins from the sea raven, Hemitripterus Americanus. future evidence for diversity among fish peptide antifreezes. J. Biol. Chem. 256, 2022-2026 (1981).

45 Gronward, W., Loewen, M. C., Lix, B., Daugulis, A. J., Sönnichsen, F. D., Davies, P. L. \& Sykes, B. D. The solution structure of type II antifreeze protein reveals a new member of the lectin family. Biochemistry 37, 4712-4721 (1998).

46 Sönnichsen, F. D., Sykes, B. D., Chao, H. \& Davies, P. L. The nonhelical structure of antifreeze protein type III. Science 259, 1154-1157 (1993)

47 Deng, G., Andrews, D. W. \& Laursen, R. A. Amino acid sequence of a new type of antifreeze protein, from the longhorn sculpin Myoxyxephalus Octodecimspinosis. FEBS Lett. 402, 17-20 (1997).

48 Graether, S. P., Kuiper, M. J., Gange, S. M., Walker, V. K., Jia, Z., Sykes, B. D. \& Davies, P. L. $\beta$-helix structure and ice-binding properties of a hyperactive antifreeze protein from an insect. Nature 406, 325-328 (2000).

49 Liou, Y., Tocilj, A., Davies, P. L. \& Jia, Z. Mimicry of ice structure by surface hydroxyls and water of a $\beta$-helix antifreeze protein. Nature 406, 322-324 (2000).

50 Schrag, J. D., O' Gray, S. M. \& DeVries, A. L. Relationship of amino acid composition and molecular weight of antifreeze glycopeptides to non-colligative freezing point depression. Biochim. Biophys. Acta. 717, 322 (1982). 
51 Uda, Y., Zepeda, S., Kaneko, F., Matsuura, Y. \& Furukawa, Y. Adsorption-induced conformational changes of antifreeze glycoproteins at the ice/water interface. J. Phys. Chem. B 111, 14355-14361 (2007).

52 Madura, J. D., Baran, K. \& Wierzbicki, A. Molecular recognition and binding of thermal hysteresis proteins to ice. J. Mo. Recognit. 13, 101-113 (2000).

53 Hobbs, P. V. in Ice Phys. (Clarendon Press, 1967).

54 Scotter, A. J., Marshall, C. B., Graham, L. A., Gilbert, J. A., Garnham, C. P. \& Davies, P. L. The basis for hyperactivity of antifreeze proteins. Cryobiology 53, 229-239 (2006).

55 Zang, W. \& Laursen, R. A. Structure-function relationships in a type I antifreeze polypeptide. The role of threonine methyl and hydroxyl groups in antifreeze activity. J. Biol. Chem. 52, 34806 (1998).

56 Baardsnes, J., Kondejewski, L. H., Hodge, R. S., Chao, H., Kay, C. \& Davies, P. L. New ice-binding face for type I antifreeze protein. FEBS Lett. 463, 87 (1999).

57 Tyshenko, M. G., Doucet, D., Davies, P. L. \& Walker, V. K. The antifreeze potential of the spruce budworm thermal hysteresis protein. Nat. Biotech. 15, 887-890 (1997).

58 Zepeda, S., Yokoyama, E., Uda, Y., Katagiri, C. \& Furukawa, Y. In situ observation of antifreeze glycorotein kinetics at the ice interface reveals atwo-step reversible adsorption mechanism. Cryst. Growth Des. 8, 3666-3672 (2008).

59 Liu, K., Jia, Z., Chen, G., Tung, C. \& Liu, R. Systematic size study of an insect antifreeze protein and its interaction with ice. Biophys. J. 88, 953-958 (2005).

60 Karim, O. A. \& Haymet, A. D. J. The ice/water interface: a molecular dynamics simulation study. J. Chem. Phys. 89, 6889-6896 (1988).

61 Beaglehole, D. \& Wilson, P. W. Thickness and anisotropy of the ice-water interface J. Phys. Chem. 97, 11053-11058 (1993).

$62 \mathrm{Nada}, \mathrm{H}$. \& Furukawa, Y. Anisotropic properties of ice/water interface: A molecular dynamics study. Jpn. J. Appl. Phys. 34, 583-588 (1995).

63 Nada, H. \& Furukawa, Y. Anisotropy in growth kinetics at interfaces between protondisordered hexagonal ice and water: a molecular dynamics study using the six-site model of $\mathrm{H}_{2} \mathrm{O}$. J. Cryst. Growth 283, 242-256 (2005).

64 Wierzbicki, A., Dalal, P., Cheatham, III T. E., Knickelbein, J. E., Haymet, A. D. J. \& Madura, J. D. Antifreeze proteins at the ice/water interface: three calculated discriminating properties for orientation of type I proteins. Biophys. J. 93, 1442-1451 (2007)

65 McDonald, S. M., White, A. \& Clancy, P. Binding of an antifreeze polypeptide to an ice/ water interface via computer simulation. AIChE. J. 41, 959-973 (1995).

66 Dalal, P., Knickelbein, J., Haymet, A. D. J., Sönnichsen, F. D. \& Madura, F. D. Hydrogen bond analysis of type 1 antifreeze protein in water and the ice/water interface. Phys. Chem .Comm. 7, 1-5 (2001).

$67 \mathrm{Nada}, \mathrm{H}$. \& van der Eerden, J. P. An intermolecular potential model for the simulation of ice and water near the melting point: a six-site model of $\mathrm{H}_{2} \mathrm{O}$. J. Chem. Phys. 118, 7401-7413 (2003).
68 Berendsen, H. J. C., Grigera, J. R. \& Straatsma, T. P. The missing term in effective pair potentials. J. Phys. Chem. 91, 6269-6271 (1987).

69 Jorgensen, W. L., Chandrasekhar, J., Madura, J. D., Impey, R. W. \& Klein, M. L. Comparison of simple potential functions for simulating liquid water. J. Chem. Phys. 79, 926-935 (1983).

70 Mahoney, M. W. \& Jorgensen, W. L. A. Five-site model for liquid water and the reproduction of density anomaly by rigid, nonpolarizable potential functions. J. Chem. Phys. 112, 8910-8922 (2000).

71 Nada, H., van der Eerden, J. P. \& Furukawa, Y. A. Clear observation of crystal growth of ice from water in a molecular dynamics simulation with a six-site potential model of $\mathrm{H}_{2}$ O. J. Cryst. Growth 266, 297-302 (2004).

72 Carignano, M. A., Shepson, P. B. \& Szleifer, I. Molecular dynamics simulations of ice growth from supercooled water. Mol. Phys. 103, 2957-2967 (2005).

$73 \mathrm{Nada}, \mathrm{H}$. Analysis of ice crystal growth shape under high pressure using molecular dynamics simulation. Cryst. Growth Des. 11, 3130-3136 (2011).

74 Haymet, A. D. J., Ward, L. G., Harding, M. M. \& Knight, C. A. Valine substituted winter flounder 'antifreeze': preservation of ice growth hysteresis. FEBS Lett. 430, 301-306 (1998).

75 Liepinsh, E., Otting, G., Harding, M. M., Ward, L. G., Mackay, J. P. \& Haymet, A. D. J. Solution structure of a hydrophobic analogue of the winter flounder antifreeze protein. Eur. J. Biochem. 269, 1259-1266 (2002).

76 Haymet, A. D. J., Ward, L. G. \& Harding, M. M. Winter flounder 'Antifreeze' proteins: synthesis and ice growth inhibition of analogues that probe the relative importance of hydrophobic and hydrogen-binding interactions. J. Am. Chem. Soc. 121, 941-948 (1999).

77 Neria, E., Fischer, S. \& Karplus, M. Simulation of activation free energies in molecular systems. J. Chem. Phys. 105, 1902-1921 (1996).

78 Nada, H. \& Furukawa, Y. in Physics and Chemistry of Ice 2010 (eds. Furukawa, Y., Sazaki, G., Uchida, T. \& Watanabe, N.) 429-436 (Hokkaido Univ. Press, Sapporo, 2011).

79 Garnham, C. P., Campbell, R. L. \& Davies, P. L. Anchored clathrate waters bind antifreeze proteins to ice. Proc. Natl. Acad. Sci. USA 108, 7363-7367 (2011).

80 Nutt, D. R. \& Smith, J. C. Dual function of the hydration layer around an antifreeze protein revealed by atomistic molecular dynamics simulations. J. Am. Chem. Soc. 130, 13066-13073 (2008).

81 Nada, H., Zepeda, S., Miura, H. \& Furukawa, Y. Significant alternations in anisotropic ice growth rate induced by the ice nucleation-active bacteria Xanthomonas Campestris. Chem. Phys. Lett. 498, 101-106 (2010).

82 Nomura, K. \& Furukawa, Y. in J. Jpn. Association for Crystal Growth (in Japanese) 31, 177 (2004).

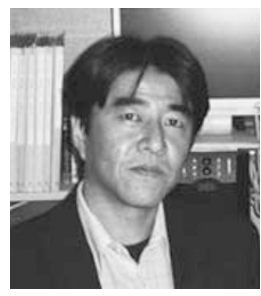

Hiroki Nada received his Bachelor degree in Applied Physics and his Master degree in Geophysics from Hokkaido University in 1991 and 1993, respectively. He received his Ph.D. from Hokkaido University under the supervision of Prof. Yoshinori Furukawa in 1995. Since 2001, he has been a senior researcher at Research Institute of Environmental Management and Technology, National Institute of Advanced Industrial Science and Technology (AIST). He received JSAP Award B in 1996 and JACG Award in 2008. His research interests include phase transition physics, crystal growth, molecular science, surface and interface, biomineralization, and physics and chemistry of ice and water.

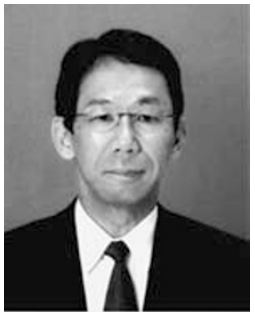

Yoshinori Furukawa received his Bachelor and Master degrees in Geophysics from Hokkaido University in 1973 and 1975, respectively. He received his Ph.D. from Hokkaido University in 1981. Since 2007, he has been the Professor of Institute of Low Temperature Science, Hokkaido University. Since 2011, he has also been the Director of Institute of Low Temperature Science, Hokkaido University. He received Award of Japanese Society of Snow and Ice in 1992 and JACG Award in 1994. His research interests include phase transition of snow and ice, crystal growth, surface and interface, and cryobiology. 